\title{
Optimal Intervention Strategies for the Spread of Obesity
}

\author{
Chunyoung $\mathrm{Oh}^{1}$ and Masud $M \mathrm{~A}^{2}$ \\ ${ }^{1}$ Department of Mathematics Education, Chonnam National University, Gwangju 500-757, Republic of Korea \\ ${ }^{2}$ Department of Mathematics, Kyungpook National University, Daegu 702-701, Republic of Korea \\ Correspondence should be addressed to Chunyoung Oh; cyoh@jnu.ac.kr
}

Received 16 March 2015; Accepted 3 June 2015

Academic Editor: Zhen Jin

Copyright (C) 2015 C. Oh and M. M A. This is an open access article distributed under the Creative Commons Attribution License, which permits unrestricted use, distribution, and reproduction in any medium, provided the original work is properly cited.

\begin{abstract}
The present study considers a deterministic compartmental model for obesity dynamics. The model exhibits forward bifurcation at basic reproduction number, $\mathscr{R}_{0}=1$, that is; for $\mathscr{R}_{0}<1$, obesity is not sustained. However for $\mathscr{R}_{0}>1$ the model approaches a locally asymptotically stable endemic equilibrium. To control this epidemic and reduce the obesity at the endemic equilibrium, we considered intervention strategies for the spread of overweight and obesity, where Pontryagin's Maximum Principle is applied. The numerical technique was used to show that there are effective control strategies that include minimizing the social contact rate with the overweight and obese population and campaigning. Numerical results indicated the effects of the two controls (prevention and education/campaigning) to be different. In societies with lower obesity, the social contact rate with the overweight and obese population plays a more prominent role in spreading obesity than lack of educational programs/campaigns. However, for societies with very high obesity burden, education/campaigning proved to be highly effective strategies. Reducing the social contact rate can result in other results such as a depression and an invasion of their individual rights. The appropriate approach to obesity is needed to lower obese societies.
\end{abstract}

\section{Introduction}

Excessive or abnormal fat gain in the body which produces risk factors for life is categorized as a spectrum of illness termed as obesity or overweight. To define obesity problem, Body Mass Index (BMI) is widely used. BMI more than 25 is considered to be hazardous to health and is labelled as overweight. Further, a person with BMI greater than 30 is considered as obese [1]. Obesity is not a cosmetic problem, but it rather has been proved to enhance the risk of lifethreatening diseases like coronary heart disease, high blood pressure, stroke, type 2 diabetes, metabolic syndrome, cancer, osteoarthritis, and many more [2]. Besides health issues, obesity has notable impact on economy, as it was identified that it is more than $\$ 215$ billion in the United States [3], which was estimated in early years to be $\$ 147$ billion [4]. As risk of severe diseases and growing economic drain are rooted into obesity, it deserves to be analyzed thoroughly.

Nowadays in many countries obesity has become a prevalent problem which is mostly overlooked at the beginning, as it happens slowly and does not cause any immediate health hazard. When someone is already in, s/he urges to recover, which is not possible overnight. Obesity has been identified as a contagious problem which is spreading over social networks [5] and was studied theoretically using epidemic models [69]. Epidemiology along with optimal control theory provides us with tools to assess the evolution of the problem through social network, identify major facts to control the epidemic, and finally and most importantly establish optimal control strategy. The authors in [6] studied infant obesity and through numerical simulation they pointed to food consumption behavior as a propeller for childhood obesity. Considering adult obesity, the authors in [7] claimed that prevention strategies are more effective by analyzing a variant of SIS epidemic model numerically. The authors in [8] experimented a system of SIS difference equation model and drew a similar conclusion. But in these studies constant controls have been considered which might not be optimal and practically unfeasible. In [9], time-dependent controls for obesity including dietary program for healthy life campaign and treatment have 
been considered in a deterministic model. It was suggested that intervention program should be implemented as early as possible to attack comparatively small epidemic, which was also proved for another social contagious problem [10].

However, nowadays in some countries obesity captured more than $70 \%$ of the total population [11, 12] and in some countries it is not so high; that is, obesity is affecting different societies in different scale. We have shown in this paper that intervention strategies for societies with different obesity burden are different. With this aim in Section 2 we presented our model. In Section 3, the dynamics of system was discussed. We pointed out the factors to be considered as intervention measures, proved the existence of optimal control strategy, and presented numerical results in Sections 4 and 5 . Conclusion has been drawn in the last section.

\section{Mathematical Model}

The model is modified from the model of [13] as follows:

$$
\begin{aligned}
\frac{d S(t)}{d t}= & \delta O(t)-\beta S(t)(O(t)+H(t)), \\
\frac{d O(t)}{d t}= & \beta S(t)(O(t)+H(t))+\eta H(t) \\
& -(\gamma+\delta) O(t), \\
\frac{d H(t)}{d t}= & \gamma O(t)-\eta H(t) .
\end{aligned}
$$

In the model of [13], the social contacts with the overweight and obese population are $\beta_{1}$ and $\beta_{2}$, respectively. However, in model (1), we used $\beta\left(=\beta_{1}=\beta_{2}\right)$; that is, we considered that both parameters are the same, as they are close enough to each other.

In this model, the adult population is divided into three subpopulations: the normal population $S(t)$, overweight population $O(t)$, and obese population $H(t)$. The adult population sizes at time $t$ are normalized to unity; that is, $S(t)+O(t)+H(t)=1,0 \leq S, O, H \leq 1$. All parameters used in the model are assumed to be strictly positive constants.

The transitions between the subpopulations $S(t), O(t)$, and $H(t)$ are governed by terms proportional to the sizes of these subpopulations. The transitions from the normal compartment to the overweight compartment occurred at a rate of $\beta$. The rate at which overweight adults with an unhealthy lifestyle and inactivity become obese individuals is $\gamma$. The obese individuals become overweight adults at a rate of $\eta$ and the overweight individuals become normal at a rate of $\delta$ due to healthy lifestyles such as activity and less food consumption. The basic reproduction number [14] $\mathscr{R}_{0}$ is given by

$$
\mathscr{R}_{0}=\frac{\beta(\eta+\gamma)}{\delta \eta} .
$$

\section{Equilibrium and Stability}

To study the dynamics of the model, taking into account $S(t)+$ $O(t)+H(t)=1$, the following reduced version is considered:

$$
\begin{aligned}
\frac{d S(t)}{d t} & =\delta(1-S(t)-H(t))-\beta S(t)(1-S(t)), \\
\frac{d H(t)}{d t} & =\gamma(1-S(t)-H(t))-\eta H(t) .
\end{aligned}
$$

Equating the right-hand sides of (3) to zero, we get obesity-free equilibrium (OFE), $E_{0}=(1,0)$, and endemic equilibrium (EE), $E^{e}=\left(S^{e}, H^{e}\right)$, where

$$
\begin{aligned}
S^{e} & =\frac{1}{\mathscr{R}_{0}}, \\
H^{e} & =\frac{\beta \gamma^{2}+\eta \gamma(\beta-\delta)}{\beta(\eta+\gamma)^{2}} .
\end{aligned}
$$

Lemma 1. System (3) admits OFE, $E_{0}=(1,0)$, which is locally asymptotically stable for $\mathscr{R}_{0} \leq 1$ and unstable if $\mathscr{R}_{0}>1$.

Proof. To check the stability, we compute the Jacobian of system (3) as

$$
J=\left(\begin{array}{cc}
-\beta+2 \beta S-\delta & -\delta \\
-\gamma & -\gamma-\eta
\end{array}\right) .
$$

At OFE, $E_{0}$, we have

$$
\begin{aligned}
& \operatorname{Tr}\left(J_{\mathrm{OFE}}\right)^{2}-4 \Delta\left(J_{\mathrm{OFE}}\right)=(\beta-\delta-\gamma+\eta)^{2}+4 \gamma(\beta+\eta) \\
& \quad>0,
\end{aligned}
$$

where $J_{\text {OFE }}$ is the Jacobian at OFE. If $\mathscr{R}_{0} \leq 1$, then $\operatorname{Tr}\left(J_{\mathrm{OFE}}\right)<$ 0 and $\Delta\left(J_{\text {OFE }}\right)>0$ and consequently OFE is a stable node. On the other hand, $\Delta\left(J_{\text {OFE }}\right)<0$ when $\mathscr{R}_{0}>1$, which makes OFE unstable.

Lemma 2. If $\mathscr{R}_{0}>1$, system (3) admits unique endemic equilibrium (EE), $E^{e}=\left(S^{e}, H^{e}\right)$, which is locally asymptotically stable for $\mathscr{R}_{0}>1$.

Proof. If $\mathscr{R}_{0}<1$, then $S^{e}>1$ which is impossible. Therefore, EE does not exist for $\mathscr{R}_{0}<1$.

At $\mathrm{EE}, E^{e}$, we have

$$
\begin{aligned}
& \operatorname{Tr}\left(J_{\mathrm{EE}}\right)^{2}-4 \Delta\left(J_{\mathrm{EE}}\right) \\
& \quad=\left(\frac{\delta \eta\left(1-\mathscr{R}_{0}\right)}{\eta+\gamma}-\frac{\delta \gamma}{\gamma+\eta}+(\eta+\gamma)\right)^{2}+4 \gamma \delta>0,
\end{aligned}
$$

where $J_{\mathrm{EE}}$ is the Jacobian at EE. If $\mathscr{R}_{0}>1$, then $\operatorname{Tr}\left(J_{\mathrm{EE}}\right)<0$ and $\Delta\left(J_{\mathrm{EE}}\right)>0$ and as a result EE is locally asymptotically stable.

Combining Lemmas 1 and 2, we observe that there exists only one OFE for $\mathscr{R}_{0} \leq 1$, which is locally asymptotically stable. For $\mathscr{R}_{0}>1$, there exists an OFE along with a unique 


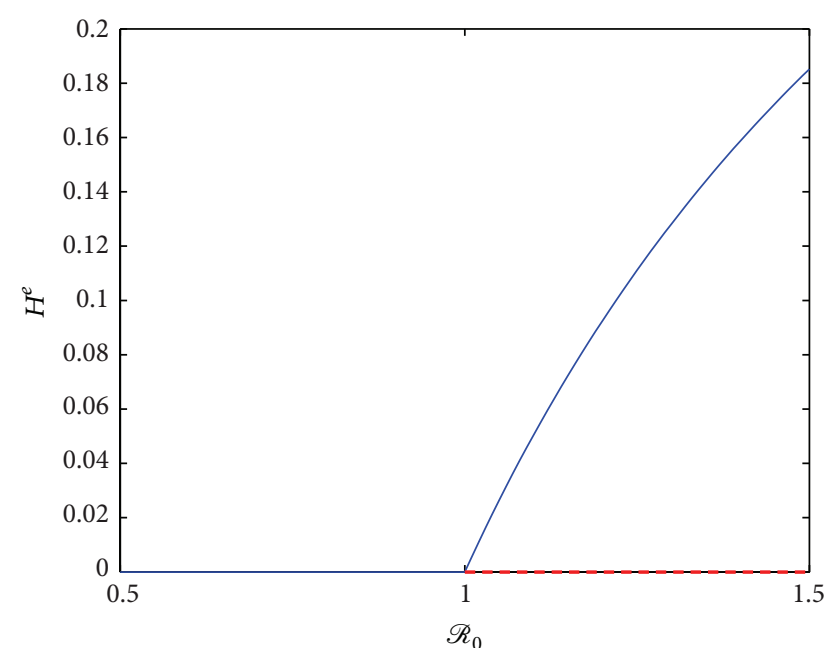

FIGURE 1: The bifurcation diagram for system (1). The solid line represents stable equilibrium and the dashed line represents unstable equilibrium.

TABLE 1: Parameters used for numerical simulation [9].

\begin{tabular}{lc}
\hline Notation & Value $\left(\right.$ week $\left.^{-1}\right)$ \\
\hline$\beta$ & 0.0007 \\
$\gamma$ & 0.00035 \\
$\delta$ & 0.00035 \\
$\eta$ & 0.00028 \\
\hline
\end{tabular}

EE between which the former is unstable while the latter is asymptotically stable. So, we can conclude that the system has a forward bifurcation at $\mathscr{R}_{0}=1$. This fact is summarized in the following theorem.

Theorem 3. System (1) exhibits a forward bifurcation at $\mathscr{R}_{0}=$ 1 .

To illustrate the bifurcation phenomena, simulations have been carried out with the parameters listed in Table 1 . The bifurcation diagram is presented in Figure 1, where the solid and the dashed lines correspond to the stable and unstable solutions, respectively. To numerically verify the existence of $\mathrm{EE}$, the phase portrait for the endemic equilibrium (EE) at $\mathscr{R}_{0}=1.5$ is shown in Figure 2, where the nearby points $(\bullet)$ of the EE approach the EE $(\diamond)$.

\section{Optimal Control}

Here, we present the sensitivity analysis of the basic reproductive number $\mathscr{R}_{0}$ :

$$
\begin{aligned}
& S_{\beta}=\frac{\partial \mathscr{R}_{0}}{\partial \beta} \frac{\beta}{\mathscr{R}_{0}}, \\
& S_{\gamma}=\frac{\partial \mathscr{R}_{0}}{\partial \gamma} \frac{\gamma}{\mathscr{R}_{0}}=\frac{\gamma}{\eta+\gamma},
\end{aligned}
$$

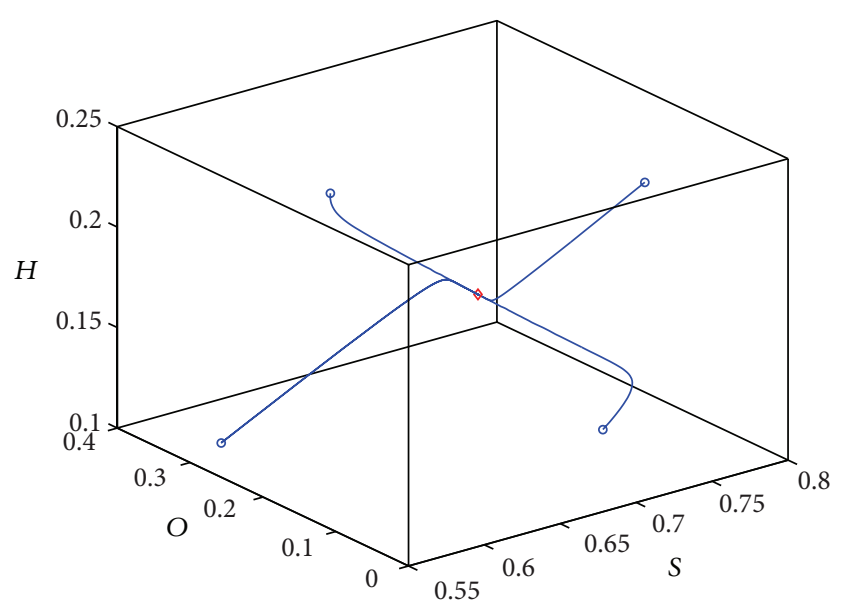

FIGURE 2: The phase portrait for the endemic equilibrium of system (1). For different initial points $(\bullet)$, the solution trajectory approaches the endemic equilibrium $(\checkmark)$ with the passage of time.

$$
\begin{aligned}
& S_{\eta}=\frac{\partial \mathscr{R}_{0}}{\partial \eta} \frac{\eta}{\mathscr{R}_{0}}=-\frac{\gamma}{\eta^{2}(\eta+\gamma)}, \\
& S_{\delta}=\frac{\partial \mathscr{R}_{0}}{\partial \delta} \frac{\gamma}{\mathscr{R}_{0}}=-\frac{1}{\delta^{2}} .
\end{aligned}
$$

Therefore, $S_{\beta}=1,0 \leq S_{\gamma} \leq 1, S_{\eta}<0$ and $S_{\delta}<0$. So, among the sensitivity indices, $S_{\beta}$ and $S_{\gamma}$ are positive, implying that the value of $\mathscr{R}_{0}$ could be reduced by reducing $\beta$ and $\gamma$. Therefore, we see that controlling $\beta$ and $\gamma$ might be a way to control the transmission of overweight and obesity. To control the prevalence rate of overweight and obesity, we expand our basic model (1), by adopting the time-dependent controls $u_{1}(t)$ and $u_{2}(t)$ corresponding to $\beta$ and $\gamma$, respectively. The control variables $1 \geq u_{1}(t) \geq 0$ and $1 \geq u_{2}(t) \geq 0$ represent the amount of intervention at time $t$ to reduce social contact with the overweight and obese population and the educational program/campaign and warning about obesity that are used to promote activity and less food consumption, respectively. In modified model (9), we replace the social contact rate $\beta$ by $\beta\left(1-u_{1}(t)\right)$ and $\gamma$ by $\gamma\left(1-u_{2}(t)\right)$. The factor $\left(1-u_{1}(t)\right)$ reduces social contacts with the overweight and obese population. The factor $\left(1-u_{2}(t)\right)$ reduces inactivity and food consumption. Now, the modified model is a nonautonomous one and results in qualitatively different output.

Our aim is to implement the intervention techniques to effectively and economically control the epidemic by implementing a healthy lifestyle that promotes exercise and less food consumption. Taking into account the above assumptions, the modified model reduces to a nonautonomous system, which is as follows:

$$
\begin{aligned}
\frac{d S(t)}{d t}= & \delta O(t) \\
& -\left[\beta\left(1-u_{1}(t)\right) S(t)(O(t)+H(t))\right],
\end{aligned}
$$




$$
\begin{aligned}
\frac{d O(t)}{d t}= & {\left[\beta\left(1-u_{1}(t)\right) S(t)(O(t)+H(t))\right]-\delta O(t) } \\
& +\eta H(t)-\gamma\left(1-u_{2}(t)\right) O(t), \\
\frac{d H(t)}{d t}= & \gamma\left(1-u_{2}(t)\right) O(t)-\eta H(t) .
\end{aligned}
$$

In the above system, $\mathscr{R}_{0}$ becomes time dependent and can be written as $\mathscr{R}_{0}(t)=\beta\left(1-u_{1}(t)\right)\left(\eta+\gamma\left(1-u_{2}(t)\right)\right) / \delta \eta$.

In the optimal control problem for ordinary differential equations, we used $u(t)$ for the control and $x(t)$ for the state. The state variable satisfies a differential equation which depends on the control variable:

$$
x^{\prime}(t)=g(t, x(t), u(t)) .
$$

The optimal control problem consists of finding a piecewise continuous control $u(t)$ and the associated state variable $x(t)$ to maximize the given objective functional; that is,

$$
\begin{aligned}
\max _{u} & \int_{0}^{T} f(t, x(t), u(t)) d t \\
\text { subject to } & x^{\prime}(t)=g(t, x(t), u(t)) \\
& x(0)=x_{0}, x(T) \text { free. }
\end{aligned}
$$

Such a maximizing control is called an optimal control [15].

Functions $f$ and $g$ will always be continuously differentiable in all three arguments. Thus, as the control will always be piecewise continuous, the associated states will always be piecewise differentiable.

Theorem 4 (see [15]). If $u^{*}(t)$ and $x^{*}(t)$ are optimal for problem (11), then there exists a piecewise differentiable adjoint variable $\lambda(t)$ such that

$$
\mathscr{H}\left(t, x^{*}(t), u(t), \lambda(t)\right) \leq \mathscr{H}\left(t, x^{*}(t), u^{*}(t), \lambda(t)\right),
$$

for all controls $u$ at each time t, where the Hamiltonian $\mathscr{H}$ is

$$
\begin{aligned}
\mathscr{H} & =f(t, x(t), u(t))+\lambda(t) g(t, x(t), u(t)), \\
\lambda^{\prime}(t) & =-\frac{\partial \mathscr{H}\left(t, x^{*}(t), u^{*}(t), \lambda(t)\right)}{\partial x}, \\
\lambda(T) & =0, \quad \text { transversality condition. }
\end{aligned}
$$

An optimal control problem with the objective cost functional can be given as

$$
\begin{aligned}
& J\left(u_{1}, u_{2}\right) \\
& =\int_{0}^{T}\left[A_{1} O(t)+A_{2} H(t)+\frac{B_{1}}{2} u_{1}^{2}(t)+\frac{B_{2}}{2} u_{2}^{2}(t)\right] d t
\end{aligned}
$$

subject to the state system given by (9).

Our goal is to show that it is possible to minimize overweight and obesity in adults and the cost of implementing the control by using possible minimal control variables $u_{i}$ for $i=1,2$.
In the objective functional, the quantities $A_{1}$ and $A_{2}$ represent the weight constants of the overweight and obese population, respectively. In the objective functional, the weight coefficients $B_{1}$ and $B_{2}$ are constants that represent cost size. The terms $(1 / 2) B_{1} u_{1}^{2}$ and $(1 / 2) B_{2} u_{2}^{2}$ describe the costs associated with the transmission by social contact rate with the overweight and obese population and an educational program/campaign and warning about obesity for minimization of the obese population, respectively.

Our aim is to find the optimal control functions $u_{1}^{*}$ and $u_{2}^{*}$. We need to find optimal control functions $\left(u_{1}^{*}, u_{2}^{*}\right)$ such that

$$
J\left(u_{1}^{*}, u_{2}^{*}\right)=\min \left\{J\left(u_{1}, u_{2}\right) \mid\left(u_{1}, u_{2}\right) \in \mathcal{U}\right\}
$$

subject to the state system given by (9), where the control set is defined as

$$
\begin{aligned}
\mathscr{U} & =\left\{\left(u_{1}, u_{2}\right) \mid u_{i}:[0, T]\right. \\
& \left.\longrightarrow[0,1], u_{i} \text { is Lebesgue measurable, } i=1,2\right\} .
\end{aligned}
$$

Let us go back to the optimal control problem (9), (14), and (15). We note that the existence of an optimal control pair can be proven.

Theorem 5. Consider control problem (14) associated with system equation (9). Then, there exists a control $\mathbf{u}^{*}=$ $\left(u_{1}^{*}, u_{2}^{*}\right) \in \mathcal{U}$ such that

$$
J\left(u_{1}^{*}, u_{2}^{*}\right)=\min _{\left(u_{1}, u_{2}\right) \in \mathscr{U}}\left\{J\left(u_{1}, u_{2}\right)\right\}
$$

with the initial conditions at $t=0$.

We seek the minimal value. To do this, we defined the Hamiltonian $\mathscr{H}$ for the control problem as follows:

$$
\begin{aligned}
\mathscr{H} & (\mathbf{X}(t), \mathcal{U}(t), \Lambda(t))=A_{1} O(t)+A_{2} H(t)+\frac{B_{1}}{2} \\
\cdot & u_{1}^{2}(t)+\frac{B_{2}}{2} u_{2}^{2}(t)+\Lambda(t)\left(\frac{d \mathbf{X}(t)}{d t}\right)^{T}=A_{1} O(t) \\
+ & A_{2} H(t)+\frac{B_{1}}{2} u_{1}^{2}(t)+\frac{B_{2}}{2} u_{2}^{2}(t)+\lambda_{1}(t)[\delta O(t) \\
- & \left.\beta\left(1-u_{1}(t)\right) S(t)(O(t)+H(t))\right]+\lambda_{2}(t) \\
\cdot & {\left[\beta\left(1-u_{1}(t)\right) S(t)(O(t)+H(t))\right.} \\
- & \left.\delta O(t)+\eta H(t)-\gamma\left(1-u_{2}(t)\right) O(t)\right]+\lambda_{3}(t) \\
\cdot & {\left[\gamma\left(1-u_{2}(t)\right) O(t)-\eta H(t)\right], }
\end{aligned}
$$

where the adjoint variable is defined by $\Lambda(t)=$ $\left(\lambda_{1}(t), \lambda_{2}(t), \lambda_{3}(t)\right)$ and the state variables for the population dynamics are denoted by $\mathbf{X}(t)=(S(t), O(t), H(t))$.

For the necessary condition of our control problem, we state and prove the following theorem.

Theorem 6. Let $S^{*}(t), O^{*}(t)$, and $H^{*}(t)$ be the optimal state solutions with the associated optimal control variables $u_{1}^{*}$ and 
$u_{2}^{*}$ for the optimal control problem (9) and (14). Then, there exist adjoint variables $\lambda_{1}(t), \lambda_{2}(t)$, and $\lambda_{3}(t)$ that satisfy

$$
\begin{aligned}
& \lambda_{1}^{\prime}(t)=-\left(\lambda_{2}(t)-\lambda_{1}(t)\right) \beta\left(1-u_{1}^{*}(t)\right) \\
& \cdot\left(O^{*}(t)+H^{*}(t)\right) \\
& \lambda_{2}^{\prime}(t)=-A_{1}\left(\lambda_{2}(t)-\lambda_{1}(t)\right) \beta\left(1-u_{1}^{*}(t)\right) S^{*}(t) \\
& \quad \cdot\left(\lambda_{3}(t)-\lambda_{2}(t)\right) \gamma\left(1-u_{2}^{*}(t)\right)+\left(\lambda_{2}(t)-\lambda_{1}(t)\right) \delta \\
& \lambda_{3}^{\prime}(t)=-A_{2}-\left(\lambda_{2}(t)-\lambda_{1}(t)\right) \beta\left(1-u_{1}^{*}(t)\right) S^{*}(t) \\
& \quad-\left(\lambda_{2}(t)-\lambda_{3}(t)\right) \eta
\end{aligned}
$$

with transversality conditions (or boundary conditions)

$$
\lambda_{j}(T)=0, \quad i=1,2,3 .
$$

Furthermore, the optimal controls $u_{1}^{*}$ and $u_{2}^{*}$ are given by

$$
\begin{aligned}
u_{1}^{*}(t) & =\min \{1, \\
\left.\max \left\{0, \frac{1}{B_{1}} \beta S^{*}(t)\left(O^{*}(t)+H^{*}(t)\right)\left(\lambda_{2}(t)-\lambda_{1}(t)\right)\right\}\right\}, & \\
u_{2}^{*}(t) & =\min \left\{1, \max \left\{0, \frac{1}{B_{2}}\left(\lambda_{3}(t)-\lambda_{2}(t)\right) \gamma O^{*}(t)\right\}\right\} .
\end{aligned}
$$

Proof. To determine the adjoint equations and the transversality conditions, we used the Hamiltonian (15). By Pontryagin's Maximum Principle, putting $S(t)=S^{*}(t), O(t)=O^{*}(t)$, and $H(t)=H^{*}(t)$ and also differentiating the Hamiltonian (15) with respect to $S(t), O(t)$, and $H(t)$, we obtain

$$
\begin{aligned}
\frac{\partial \mathscr{H}}{\partial S} & \\
= & \left(\lambda_{2}(t)-\lambda_{1}(t)\right) \beta\left(1-u_{1}^{*}(t)\right)\left(O^{*}(t)+H^{*}(t)\right), \\
\frac{\partial \mathscr{H}}{\partial O} & \\
= & A_{1}+\left(\lambda_{2}(t)-\lambda_{1}(t)\right) \beta\left(1-u_{1}^{*}(t)\right) S^{*}(t) \\
& +\left(\lambda_{3}(t)-\lambda_{2}(t)\right) \gamma\left(1-u_{2}^{*}(t)\right) \\
& -\left(\lambda_{2}(t)-\lambda_{1}(t)\right) \delta, \\
\frac{\partial \mathscr{H}}{\partial H} & \\
= & A_{2}+\left(\lambda_{2}(t)-\lambda_{1}(t)\right) \beta\left(1-u_{1}^{*}(t)\right) S^{*}(t) \\
& +\left(\lambda_{2}(t)-\lambda_{3}(t)\right) \eta .
\end{aligned}
$$

The costate equations are

$$
\begin{aligned}
& \lambda_{1}^{\prime}(t)=-\frac{\partial \mathscr{H}}{\partial S}, \\
& \lambda_{2}^{\prime}(t)=-\frac{\partial \mathscr{H}}{\partial O}, \\
& \lambda_{3}^{\prime}(t)=-\frac{\partial \mathscr{H}}{\partial H} .
\end{aligned}
$$

To obtain the optimality conditions (21), we also differentiated the Hamiltonian $\mathscr{H}$ with respect to $u_{1}, u_{2}$ and set it equal to zero:

$$
\begin{aligned}
0= & \frac{\partial \mathscr{H}}{\partial u_{1}^{*}} \\
= & B_{1} u_{1}^{*}(t) \\
& +\left(\lambda_{1}(t)-\lambda_{2}(t)\right) \beta S^{*}(t)\left(O^{*}(t)+H^{*}(t)\right), \\
0= & \frac{\partial \mathscr{H}}{\partial u_{2}^{*}}=B_{2} u_{2}^{*}(t)+\left(\lambda_{2}(t)-\lambda_{3}(t)\right) \gamma O^{*}(t) .
\end{aligned}
$$

Solving for the optimal controls, we obtain

$$
\begin{aligned}
& u_{1}^{*}(t)=\frac{1}{B_{1}} \beta S^{*}(t)\left(O^{*}(t)+H^{*}(t)\right)\left(\lambda_{2}(t)-\lambda_{1}(t)\right), \\
& u_{2}^{*}(t)=\frac{1}{B_{2}}\left(\lambda_{3}(t)-\lambda_{2}(t)\right) \gamma O^{*}(t) .
\end{aligned}
$$

To determine an explicit expression for the optimal controls for $0 \leq u_{1}^{*} \leq 1$ and $0 \leq u_{2}^{*} \leq 1$, we utilize a standard optimality technique. We considered the following three cases:

(i) On the set $\left\{t \mid 0<u_{1}^{*}(t)<1\right\}, \quad \frac{\partial \mathscr{H}}{\partial u_{1}^{*}}=0$

$u_{1}^{*}(t)=\frac{1}{B_{1}} \beta S^{*}(t)\left(O^{*}(t)+H^{*}(t)\right)\left(\lambda_{2}(t)-\lambda_{1}(t)\right)$

(ii) On the set $\left\{t \mid u_{1}^{*}(t)=0\right\}, \quad \frac{\partial \mathscr{H}}{\partial u_{1}^{*}} \geq 0$

$\frac{1}{B_{1}} \beta S^{*}(t)\left(O(t)+H^{*}(t)\right)\left(\lambda_{2}(t)-\lambda_{1}(t)\right) \leq 0$

$$
=u_{1}^{*}(t)
$$

(iii) On the set $\left\{t \mid u_{1}^{*}(t)=1\right\}, \quad \frac{\partial \mathscr{H}}{\partial u_{1}^{*}} \leq 0$

$\frac{1}{B_{1}} \beta S^{*}(t)\left(O^{*}(t)+H^{*}(t)\right)\left(\lambda_{2}(t)-\lambda_{1}(t)\right) \geq u_{1}^{*}(t)$

$=1$. 
Combining the three cases above, we found a characterization of $u_{1}^{*}$ :

$$
\begin{aligned}
u_{1}^{*}(t) & =\min \{1, \\
& \left.\max \left\{0, \frac{1}{B_{1}} \beta S^{*}(t)\left(O^{*}(t)+H^{*}(t)\right)\left(\lambda_{2}(t)-\lambda_{1}(t)\right)\right\}\right\} .
\end{aligned}
$$

Using the same arguments, we also obtained the second optimal control function

$$
\begin{aligned}
& u_{2}^{*}(t) \\
& \quad=\min \left\{1, \max \left\{0, \frac{1}{B_{2}}\left(\lambda_{3}(t)-\lambda_{2}(t)\right) \gamma O^{*}(t)\right\}\right\} .
\end{aligned}
$$

\section{Numerical Results}

Using Pontryagin's Maximum Principle [16], the control system is determined along with the necessary conditions for the existence of an optimal control. Using the forward-backward sweep method [17], we numerically solve the nonautonomous optimality system. The optimal solutions are obtained by the iterative method. The state system with the guessed controls over the whole time is solved using the forward scheme. Then, the adjoint system with the transversality conditions is solved using the backward scheme. The previous controls and the current state variables from the first step are used in this step. The controls are updated using a convex combination of the previous controls and control values from the optimality equations obtained from the current state and adjoint variables. This iteration is repeated until the differences of the unknown values between the previous and current iterations are sufficiently close.

In this section, intervention strategies on the spread of overweight and obesity are used to propose various control scenarios using optimal control theory. The optimality system consists of three state equations (9) with initial conditions at $t=0$, three adjoint equations (19) with the transversality conditions (20), and optimality equations (21).

We simulated different scenarios for minimizing the overweight and obese adults in the model. All parameter values used in the numerical simulations were estimated based on the article [9] as given in Table 1.

Since the controls could not be sufficiently effective and depend on a person's tolerance, the upper bounds of $u_{1}^{*}$ and $u_{2}^{*}$ were chosen to be 0.6 . As an obese individual is more deteriorating to society than an overweight individual, we choose $A_{1}=1000$ and $A_{2}=2000$.

Figure 3 shows successful control of the obesity epidemic with the initial condition $S(0)=0.8, O(0)=0.15$, and $H(0)=$ 0.05 , where $O$ and $H$ are remarkably less than those in the without control case due to the implementation of $u_{1}^{*}(t)$ and $u_{2}^{*}(t)$. Besides, as an effect of the controls, $\mathscr{R}_{0}$ is reduced to as low as 1.3548 in the beginning, and we name this as $\mathscr{R}_{0, \text { min }}$. $u_{1}^{*}$ is implemented with full strength from the beginning for a long time, whereas $u_{2}^{*}(0)=0.4452$ and it decreases with time. We name this maximum implementation of $u_{1}^{*}$ and $u_{2}^{*}$ as $u_{1, \max }$ and $u_{2 \text {,max }}$, respectively, which gives us a brief idea about the control scenario. As $u_{1}^{*}$ and $u_{2}^{*}$ decrease with time $\mathscr{R}_{0}$ increases, but the fraction of overweight and obese individuals remain satisfactorily low. Consider

$$
\begin{aligned}
u_{1, \max } & :=\max \left\{u_{1}^{*}(t): 0 \leq t \leq T\right\}, \\
u_{2, \max } & :=\max \left\{u_{2}^{*}(t): 0 \leq t \leq T\right\}, \\
\mathscr{R}_{0, \min } & :=\min \left\{\mathscr{R}_{0}(t): 0 \leq t \leq T\right\} .
\end{aligned}
$$

However, the control scenario is not the same in all the cases; it varies with the cost of the controls and even with the initial conditions. The first two graphs in Figure 4 illustrate the variation of $u_{1 \text {,max }}$ and $u_{2 \text {,max }}$ for a wide range of values of the weights $B_{1}$ and $B_{2}$, respectively, and the last one plots $\mathscr{R}_{0, \min }$ against $B_{1}$ and $B_{2} . u_{1, \max }$ and $u_{2 \text {, max }}$ fall with the increase of $B_{1}$ and $B_{2}$, respectively. $\mathscr{R}_{0}$ is decreased to the lowest when $B_{1}$ and $B_{2}$ are low; that is, $u_{1 \text {,max }}$ and $u_{2 \text {,max }}$ are high and vice versa. It is conspicuous that $u_{1}$ is implemented more than $u_{2}$, which agrees with sensitivity analysis as $S_{\beta}>$ $S_{\gamma}$. But it depends on the initial condition. At this point, Figure 5 gives a clear picture. The obesity burden varies from society to society depending on the socioeconomic status, lifestyle, and food habit; for example, $70 \%$ of Mexican adults are overweight [11]; Nauru, a small island in the South Pacific, has overweight people as high as $71.1 \%$ [12]. The optimal control scenarios for different obesity burdens are plotted in Figure 5. For a low obesity burden, $u_{1}$ is used as a more effective control than $u_{2}$. Implementation of both $u_{1}$ and $u_{2}$ is higher for a higher initial obesity burden. When obesity is above $60 \%$, the maximum $u_{2}$ comes into effect. In the case of very high obesity near $75 \%$, the use of $u_{2}$ exceeds that of $u_{1}$.

\section{Conclusion and Discussion}

An autonomous system of ODE presenting obesity dynamics is considered, which exhibits forward bifurcation at $\mathscr{R}_{0}$; that is, obesity will not persist, if $\mathscr{R}_{0}<1$. However, for $\mathscr{R}_{0}>1$ in any society, obesity will be sustained, through reaching a stable state holding the burden of obesity. To control obesity in such cases, we define a nonautonomous system incorporating time-dependent control, and we set up an optimal control problem, using Pontryagin's Maximum Principle. The time-dependent optimal control reduces the number of overweight people along with the value of $\mathscr{R}_{0}$ and thus controls the obesity.

In this study, we used only two controls regarding reducing the contact rate and educational programs/campaigns. Numerical results indicated the effects of the two controls (prevention and education/campaigning) to be different. In societies with lower obesity, the social contact rate with the overweight and obese population plays a more prominent role in spreading obesity than lack of educational programs/campaigns. However, for societies with very high obesity burden, education/campaigning proved to be highly effective strategies.

Notwithstanding the efficacy and sophistication of the mathematics, reducing the social contact rate can result in 

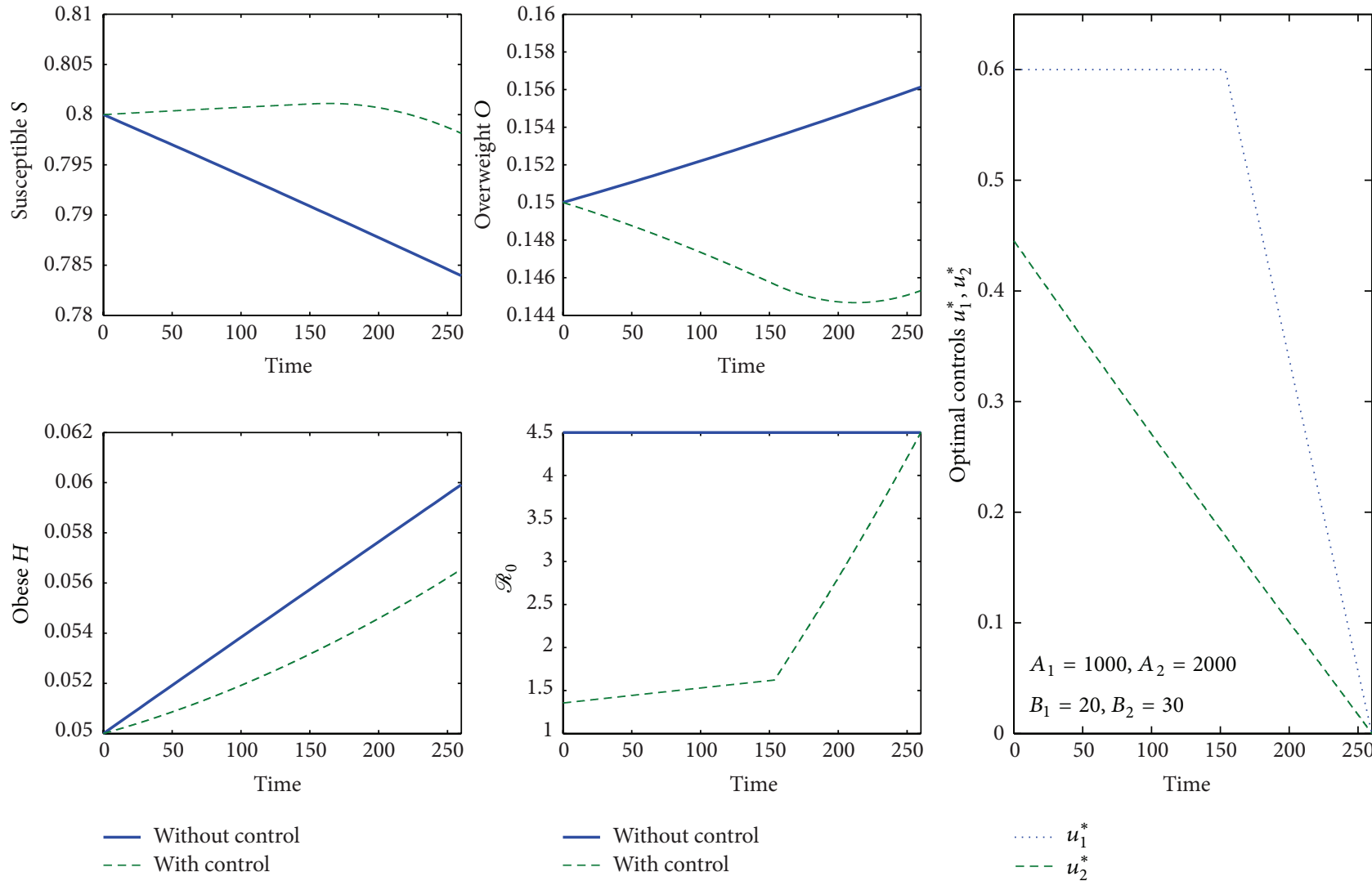

FIGURE 3: Implementation of optimal control and corresponding state of the system with initial condition $S(0)=0.8, O(0)=0.15$, and $H(0)=0.05$
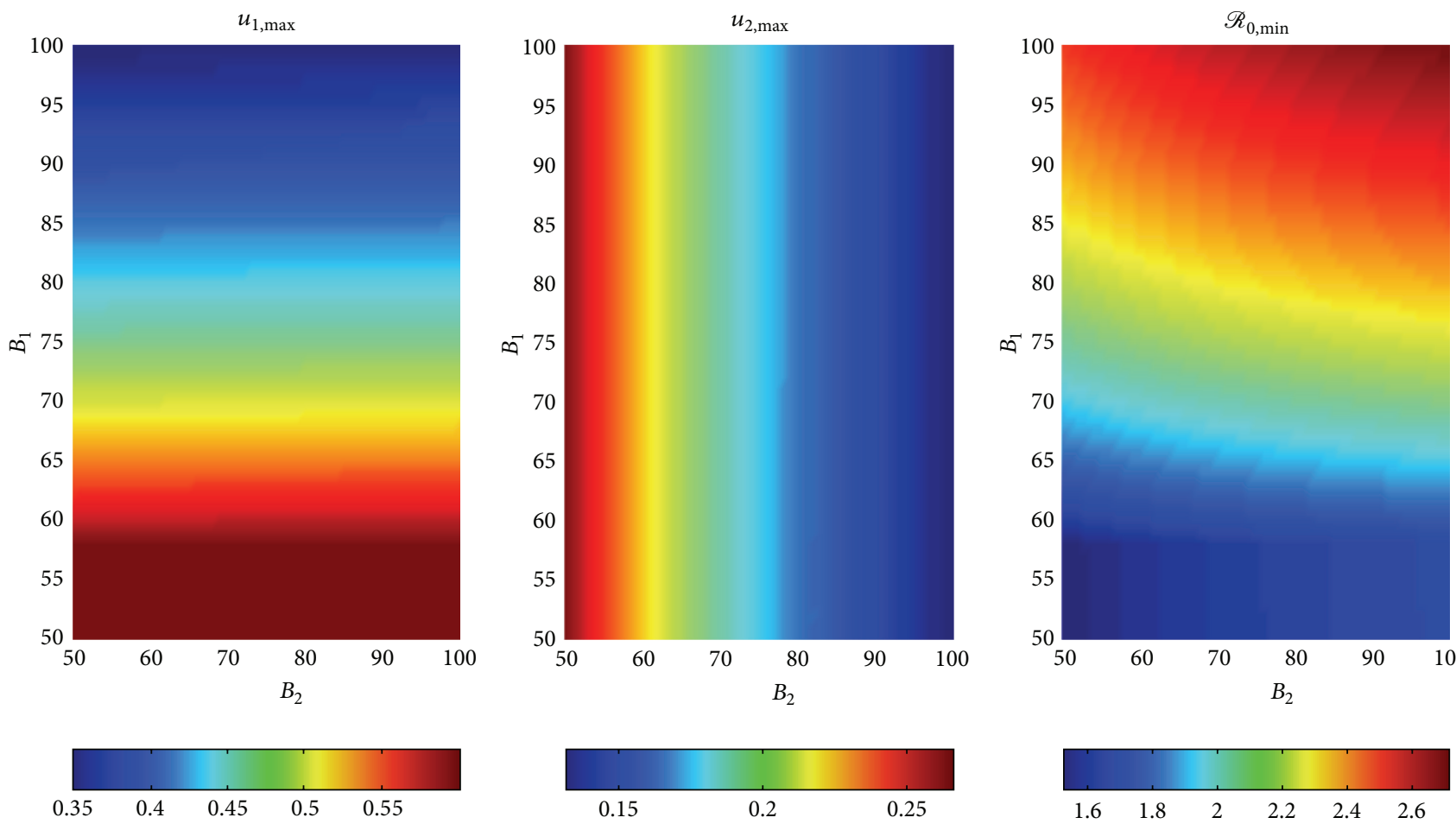

Figure 4: Maximum implementation of optimal control for different associated cost and minimum reduced level of $\mathscr{R}_{0}$ due to the implementation of optimal control. 

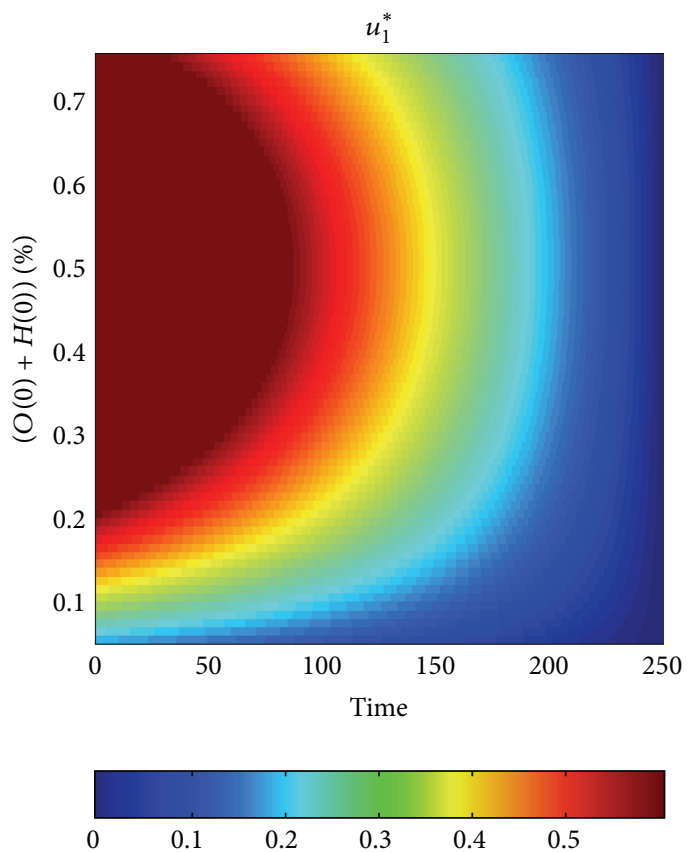


FIGURE 5: Optimal control scenario for wide range of initial obesity burden with $B_{1}=50$ and $B_{2}=50$.

other results such as a depression and an invasion of their individual rights. Therefore, appropriate approach to obesity, such as the road system facilitated for walking, is needed to lower obesity. It is important to consider the program that works best with diet and the other social systems.

\section{Conflict of Interests}

The authors declare that there is no conflict of interests regarding the publication of this paper.

\section{Acknowledgment}

This study was financially supported by Chonnam National University, 2015.

\section{References}

[1] WHO, Health topics, Obesity, http://www.who.int/topics/ obesity/en/.

[2] http://www.nhlbi.nih.gov/health/health-topics/topics/obe/ risks.

[3] R. A. Hammond and R. Levine, "The economic impact of obesity in the United States," Diabetes, Metabolic Syndrome and Obesity: Targets and Therapy, vol. 3, pp. 285-295, 2010.

[4] E. A. Finkelstein, J. G. Trogdon, J. W. Cohen, and W. Dietz, "Annual medical spending attributable to obesity: payer-and service-specific estimates," Health Affairs, vol. 28, no. 5, pp. w822-w831, 2009.

[5] N. A. Christakis and J. H. Fowler, "The spread of obesity in a large social network over 32 years," The New England Journal of Medicine, vol. 357, no. 4, pp. 370-379, 2007.
[6] L. Jódar, F. J. Santonja, and G. González-Parra, "Modeling dynamics of infant obesity in the region of Valencia, Spain," Computers \& Mathematics with Applications, vol. 56, no. 3, pp. 679-689, 2008.

[7] F.-J. Santonja, R.-J. Villanueva, L. Jódar, and G. GonzalezParra, "Mathematical modelling of social obesity epidemic in the region of Valencia, Spain," Mathematical and Computer Modelling of Dynamical Systems, vol. 16, no. 1, pp. 23-34, 2010.

[8] M. S. Kim, C. Chu, and Y. Kim, "A note on obesity as epidemic in Korea," Osong Public Health and Research Perspectives, vol. 2, no. 2, pp. 135-140, 2011.

[9] D. Aldila, N. Rarasati, N. Nuraini, and E. Soewono, "Optimal control problem of treatment for obesity in a closed population," International Journal of Mathematics and Mathematical Sciences, vol. 2014, Article ID 273037, 7 pages, 2014.

[10] B. N. Kim, M. A. Masud, and Y. Kim, "Optimal implementation of intervention to control the self-harm epidemic," Osong Public Health and Research Perspectives, vol. 5, no. 6, pp. 315-323, 2014.

[11] J. Daniel, "How Mexico got so fat and is now more obese than America," 2014, http://www.dailymail.co.uk/news/article2358472/How-Mexico-got-fat-obese-America.html.

[12] America Is No Longer the Most Obese Country in the World, http://gawker.com/America-is-no-longer-the-most-obesecountry-in-the-world-714151306.

[13] C. Oh, "A note on the obesity as an epidemic," Honam Mathematical Journal, vol. 36, no. 1, pp. 131-139, 2014.

[14] P. van den Driessche and J. Watmough, "Reproduction numbers and sub-threshold endemic equilibria for compartmental models of disease transmission," Mathematical Biosciences, vol. 180, pp. 29-48, 2002.

[15] S. Lenhart and J. T. Workman, Optimal Control Applied to Biological Models, Chapman \& Hall, CRC Press, 2007. 
[16] L. S. Pontryagin, V. G. Boltanskii, R. V. Gamkrelidze, and E. F. Mishchenko, The Mathematical Theory of Optimal Processes, Wiley, New York, NY, USA, 1962.

[17] S. Lenhart and J. T. Workman, Optimal Control Applied to Biological Models, Chapman and Hall/CRC, 2007. 


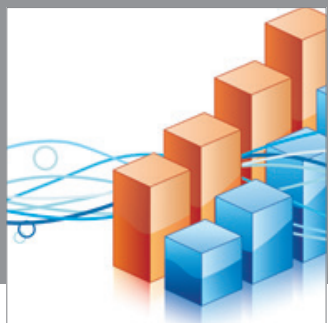

Advances in

Operations Research

mansans

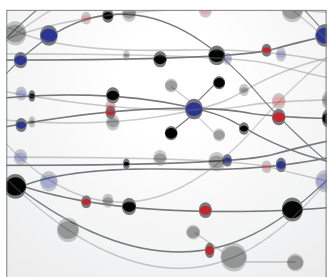

The Scientific World Journal
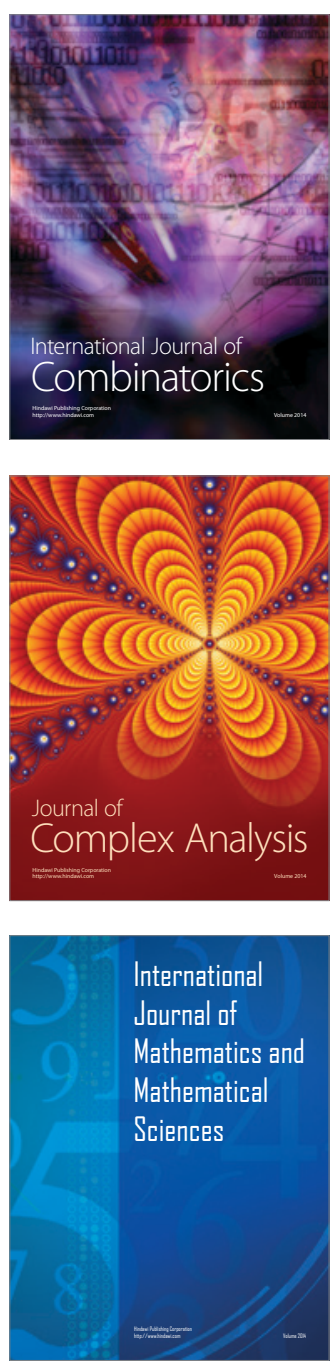
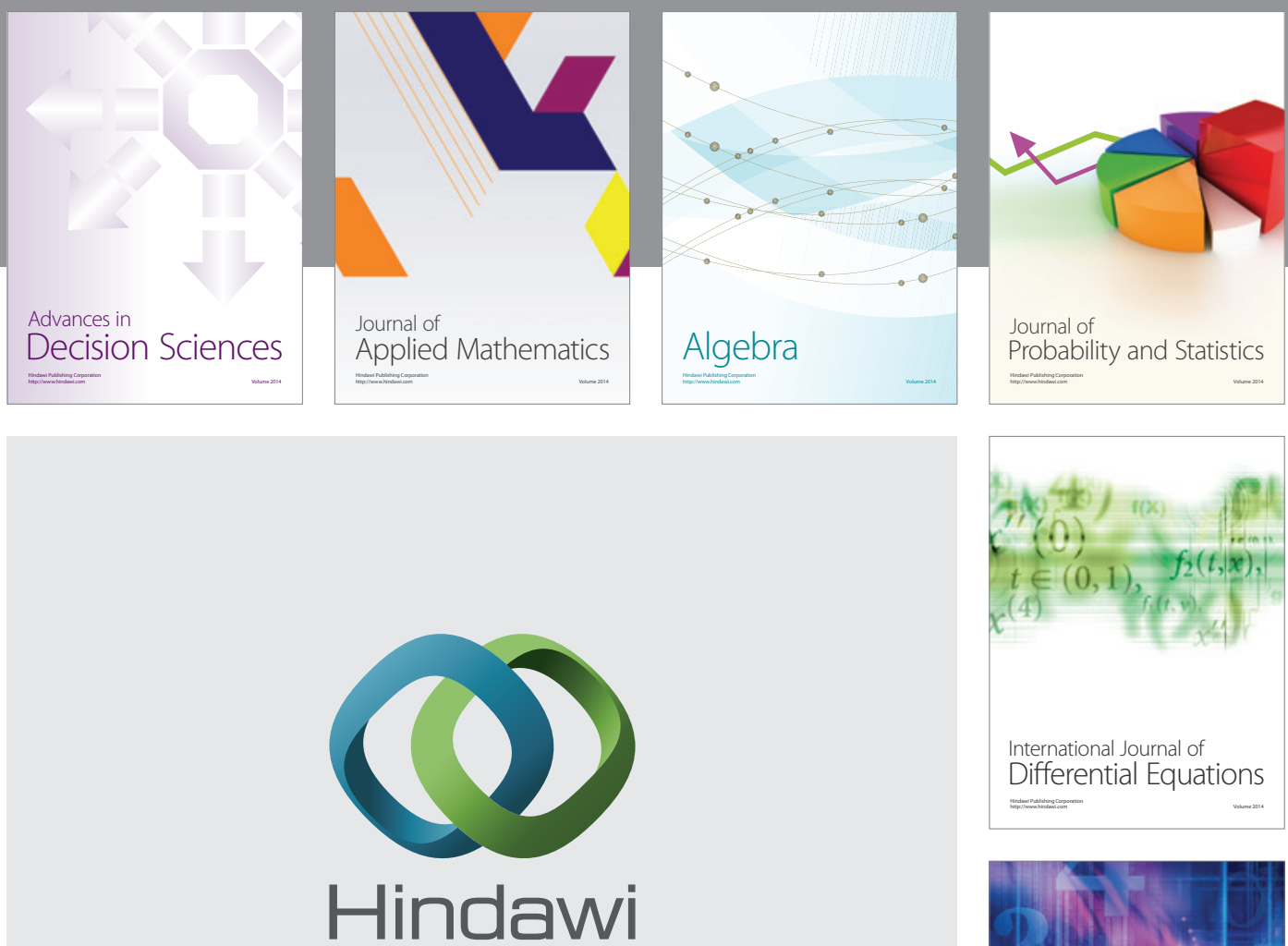

Submit your manuscripts at http://www.hindawi.com
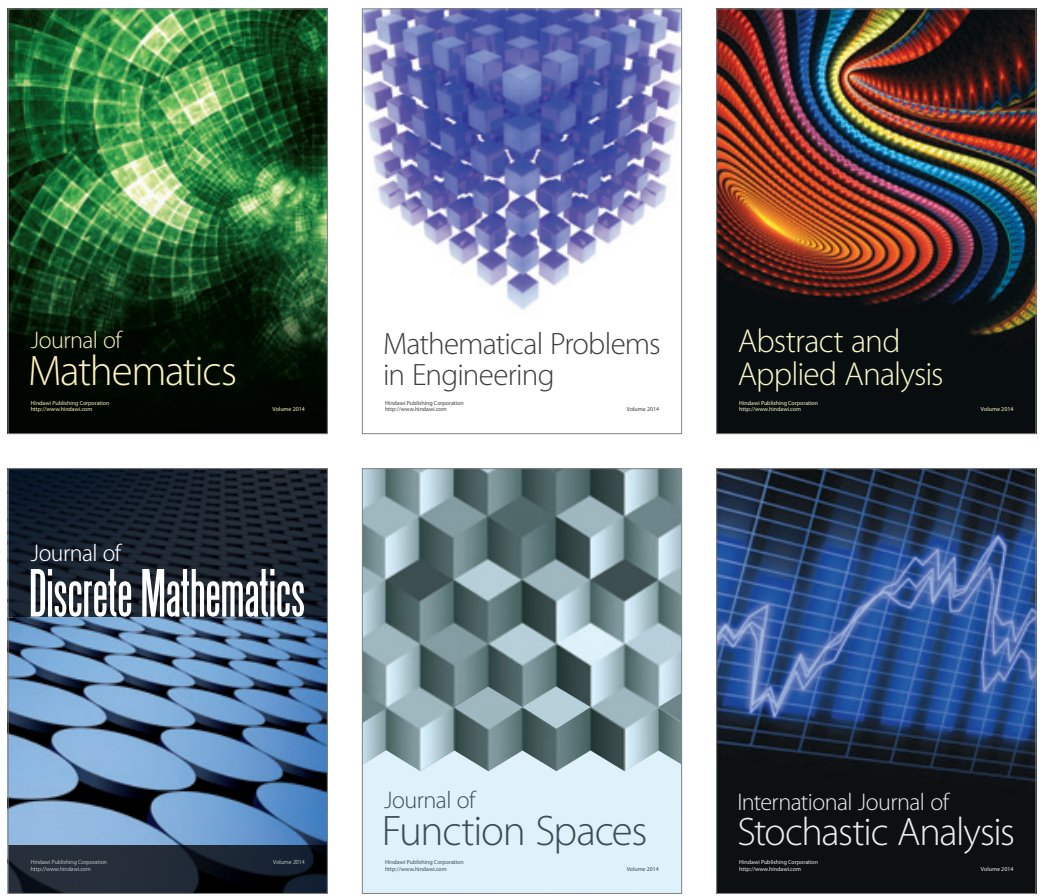

Journal of

Function Spaces

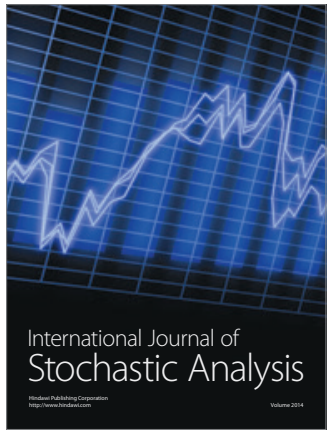

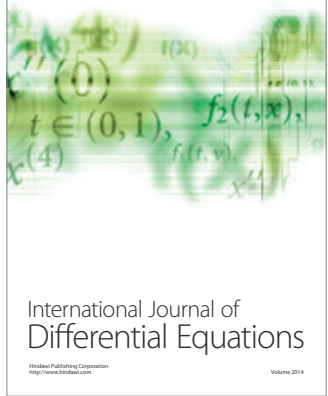
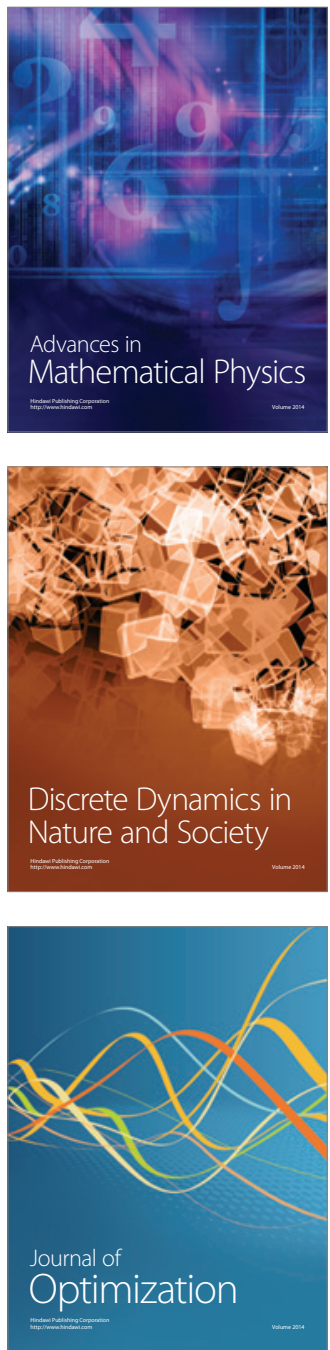